\title{
Towards Process-Driven Mobile Data Collection Applications: Requirements, Challenges, Lessons Learned
}

\author{
Johannes Schobel, Marc Schickler, Rüdiger Pryss, Fabian Maier, and Manfred Reichert \\ Institute of Databases and Information Systems, University of Ulm, James-Franck-Ring, Ulm, Germany \\ \{johannes.schobel, marc.schickler, ruediger.pryss, fabian.maier, manfred.reichert\}@uni-ulm.de
}

Keywords: Process-Aware Information System, Electronic Questionnaire, Mobile Business Application.

\begin{abstract}
In application domains like healthcare, psychology and e-learning, data collection is based on specifically tailored paper \& pencil questionnaires. Usually, such a paper-based data collection is accomplished by a massive workload regarding the processing, analysis, and evaluation of the data collected. To relieve domain experts from these manual tasks and to increase the efficiency of the data collection process, we developed a generic approach for realizing process-driven smart mobile device applications based on process management technology. According to this approach, the logic of a questionnaire is described in terms of an explicit process model whose enactment is driven by a generic process engine. Our goal is to demonstrate that such a process-aware design of mobile business applications is useful with respect to mobile data collection. Hence, we developed a generic architecture comprising the main components of mobile data collection applications. Furthermore, we used these components for developing mobile electronic questionnaires for psychological studies. The paper presents the challenges identified in this context and discusses the lessons learned. Overall, process management technology offers promising perspectives for developing mobile business applications at a high level of abstraction.
\end{abstract}

\section{INTRODUCTION}

Recently, smart mobile applications have been increasingly used in a business context. Examples include simple applications (e.g., task management), but also sophisticated analytic business applications. In particular, smart mobile devices can be used for enabling flexible mobile data collection as well (Pryss et al., 2013). Thereby, data can be collected with sensors (e.g., pulse sensor), communicating with the smart mobile device (Schobel et al., 2013), or with smart form-based applications (Pryss et al., 2012). Examples of applications requiring such a mobile data collection include clinical trials, psychological studies, and quality management surveys.

Developing a mobile data collection application requires specific knowledge on how to implement smart mobile applications. Furthermore, it requires domain-specific knowledge, usually not available to the programmers of these applications. Hence, to avoid a gap between business needs and IT solutions, continuous and costly communication between domain and IT experts becomes necessary. To improve this situation, a framework for rapidly developing and evolving mobile data collection applications is indis- pensable. In particular, respective business applications should be easy to maintain for non-computer (i.e., domain) experts as well. Our overall vision is to enable domain experts to develop mobile data collection applications at a high level of abstraction. Specifically, this paper focuses on the process-driven design, implementation, and enactment of mobile questionnaire applications, which support domain experts with their daily data collection tasks.

As application domain for demonstrating the benefits of our approach we choose psychological studies. Here, domain experts mostly use paper-based questionnaires for collecting required data from subjects. However, such a paper-based data collection shows several drawbacks, e.g., regarding the structure and layout of a questionnaire (e.g., questions may still be answered, even if they are no longer relevant or needed), or the later analysis of the answers (e.g., errors might occur when transferring the collected paper-based data to electronic worksheets).

To cope with these issues and to understand the subtle differences between paper-based and electronic questionnaires in a mobile context, first of all, we implemented several questionnaire applications for smart mobile devices and applied them in real and 
sophisticated application settings (Liebrecht, 2012; Schindler, 2013). In particular, we were able to demonstrate that electronic questionnaires relieve domain experts from costly manual tasks, like the transfer, transformation and analysis of the collected data. As a major drawback, the first applications we had implemented were hard-coded and required considerable communication with domain experts. As a consequence, these applications were neither easy to maintain nor extensible. However, in order to avoid a gap between the domain-specific design of a questionnaire and its technical implementation enacted on smart mobile devices, an easy to handle, flexible and generic questionnaire system is indispensable.

From the insights we gained during the practical use of the above mentioned mobile applications as well as from lessons learned when implementing other kinds of mobile applications (Robecke et al., 2011), we elicited the requirements for electronic questionnaire applications that enable a flexible mobile data collection. In order to evaluate whether the use of process management technology contributes to the satisfaction of these requirements, we mapped the logic of a complex questionnaire from psychology to a process model, which was deployed to a process engine. It then served as basis for driving the execution of questionnaire instances at realtime. In particular, this mapping allows us to overcome many of the problems known from paper-based questionnaires. In turn, the use of a process modeling component as well as a process execution engine in the given context, raised additional challenges, e.g., related to the process-driven execution of electronic questionnaires on and the mobile data collection with smart mobile devices. The implemented questionnaire runs on a mobile device and communicates with a remote process engine to enact psychological questionnaires. As a major lesson, we learned that process management technology may not only be applied in the context of business process automation, but also provides a promising approach for generating mobile data collection applications. In particular, a process-driven approach enables non-computer experts to develop electronic questionnaires for smart mobile devices as well as to deploy them on respective devices in order to collect data with them. lows:

In detail, the contributions of this paper are as fol-

- We discuss fundamental problems of paper-based questionnaires and present requirements regarding their transfer to smart mobile devices.

- We provide a mental model for mapping questionnaires to process models. Further, we illustrate this mental model through a real-world applica- tion scenario from the psychological domain.

- We present a generic architecture for applications running on smart mobile devices that can be used to model, visualize and enact electronic questionnaires. This approach relies on the provided mental model and uses process models to define and control the flow logic of a questionnaire.

- We share fundamental insights we gathered during the process of implementing and evaluating the mobile data collection application.

The remainder of this paper is structured as follows: Section 2 discusses issues related to paperbased questionnaires. Further, it elicitates the requirements that emerge when transferring a paperbased questionnaire to an electronic version running on smart mobile devices. Section 3 describes the mental model we suggest for meeting these requirements. In Section 4, we present the basic architecture of our approach for developing mobile data collection applications. Section 5 provides a detailed discussion, while Section 6 presents related work. Finally, Section 7 concludes the paper with a summary and outlook.

\section{CASE STUDY}

In a case study with 10 domain experts, we analyzed more than 15 paper-based questionnaires from different domains, particularly questionnaires used in the context of psychological studies. Our goal was to understand the issues that emerge when transferring paper-based questionnaires to smart mobile devices. Section 2.1 discusses fundamental issues related to paper \& pencil questionnaires. Then, Section 2.2 elicitates fundamental requirements for their electronic mobile support.

\subsection{Paper-Based Questionnaires}

We analyzed 15 paper-based questionnaires from psychology and medicine. In this context, a variety of issues emerged. First, in the considered domains, a questionnaire must be valid. This means that it should have already been applied in several studies, and statistical evaluations have proven that the results obtained from the collected data are representive. In addition, the questions are usually presented in a neutral way in order to not affect or influence the subject (e.g., patient). Creating a valid instrument is one of the main goals when setting up a psychological questionnaire. In particular, reproducible and conclusive 
results must be guaranteed. Furthermore, a questionnaire may be used in two different modes. In the interview mode, the subject is interviewed by a supervisor who also fills in the questionnaire; i.e., the supervisor controls which questions he is going to ask or skip. This mode usually requires a lot of experience since the interviewer must also deal with questions that might be critical for the subject. The other mode we consider is self-rating. In this mode, the questionnaire is handed out to the subject who then answers the respective questions herself; i.e., no supervision is provided in this mode

Another challenging issue of paper-based questionnaires concerns the analysis of the data collected. Gathered answers need to be transfered to electronic worksheets, which constitutes a time-consuming and error-prone task. In particular, note that during the interviews or the self-filling of a questionnaire, typographical errors or wrong interpretations of given answers might occur. In general, both sources of error (i.e., errors occuring during the interviews and errors occuring during the transcription) decrease the quality of the data collected, which further underlines the need of an electronic support for flexible and mobile data collection.

In numerous interviews we conducted with 10 domain experts from psychology, additional issues have emerged. Psychological studies are often performed in developing countries, e.g., surveying of child soldiers in rural areas in Africa (Crombach et al., 2013; Liebrecht, 2012). Political restrictions regarding data collection further require attention and influence the way in which interviews and assessments may be performed by domain experts (i.e., psychologists). Since in many geographic regions the available infrastructure is not well developed, data collected with paper-based questionnaires is usually digitalized in the home country of the scientists responsible for the study. Taking these issues into account, it is not surprising that psychological studies last from several weeks up to several months. From a practical point of view, this raises the problem of allocating enough space in luggage to transfer the paper-based questionnaires safely to the home country of the respective researcher.

Apart from these logistic problems, we revealed issues related to the interview procedure itself. In particular, it has turned out that questionnaires must often be adapted to a particular application context (e.g., changing the language of a questionnaire or adding / deleting selected questions). Such adaptations (by authorized domain experts) must be propagated to all other interviewers and smart mobile devices respectively in order to keep the results valid and compara- ble.

Considering these issues, we had additional discussions with domain experts from psychology, which revealed several requirements discussed in the next section.

\subsection{Requirements}

In the following, we discuss basic requirements for the mobile support of electronic questionnaires. We derived these requirements in the context of case studies, literature analyses, expert interviews, and handson experiences regarding the implementation of mobile data collection applications (Crombach et al., 2013; Ruf-Leuschner et al., 2013; Isele et al., 2013). Especially, when interviewing domain experts, fundamental requirements could be elicitated. The same applies to the various paper $\&$ pencil questionnaires we analyzed.

The major requirements are as follows:

R1 (Mobility). The process of collecting data should be highly flexible and usually requires extensive interactions. Data may have to be collected even though no PC is available at the place the questionnaire should be filled in. For example, consider data collection at the bedside of a patient in a hospital or interviews conducted by psychologists in a meeting room. PCs are often disturbing in such situations, particularly if the interviewer is "hiding" himself behind a screen. To enable flexible data collection, the device needs to be portable instead. Further, it should not distract the participating actors in communicating and interacting with each other.

R2 (Multi-User Support). Since different users may interact with a mobile questionnaire, multi-user support is crucial. In addition, it must be possible to distinguish between different user roles (e.g., interviewers and subjects) involved in the processing of an electronic questionnaire. Finally, a particular user may possess different roles. For example, an actor could be interviewer in the context of a specific questionnaire, but subject in the context of another one.

R3 (Support of Different Questionnaire Modes). Generally, a questionnaire may be used in two different modes: interview and self-rating mode (cf. Section 2.1). These two modes of questioning diverge in the way the questions are posed, the possible answers that may be given, the order in which the questions are answered, and the additional features provided (e.g., freetext notes). In general, mobile electronic questionnaire applications should allow for both modes. Note, that 
this requirement is correlated with $\mathrm{R} 2$ as the considered roles determine the modes available for a questionnaire.

R4 (Multi-Language Support). The contents of a questionnaire (e.g., questions and field labels) may have to be displayed in different languages (e.g., when conducting a psychological study globally in different countries). The actor accessing the questionnaire should be allowed to choose among several languages.

R5 (Skeuomorphism and Paper-based Style). To foster the comprehensibility of an electronic questionnaire and to ensure its validity, the latter should be designed in the same style (i.e., same order and structure) as the corresponding paperbased version. For example, this means, that the structuring of a questionnaire in different pages must be kept.

R6 (Native Application Style). Any mobile support of electronic questionnaire application must consider different mobile operating systems (e.g., Android or iOS). In this context, standard control elements of the respective mobile operating system should be used to ensure familiarity of users with the elements of the questionnaire when running the latter on their preferred smart mobile device.

R7 (Self-Explaning User Interface). The user interface should be easy to understand and provide intuitive interaction facilities. Furthermore, users should be guided through the process of collecting data with their smart mobile devices.

R8 (Maintainability). Questionnaires evolve over time and hence may have to be changed occasionally. Therefore, it should be possible to quickly and easily change the structure and content of an electronic questionnaire; e.g., to add a question, to edit the text of a question, to delete a question, or to change the order of questions. In particular, no programming skills should be required in this context; i.e., domain experts (e.g., psychologists) should be able to introduce respective changes at a high level of abstraction.

Especially, requirement R8 constitutes a major challenge, which necessitates a high level of abstraction when defining and changing electronic questionnaires, which may then be enacted on a variety of smart mobile devices. To cope with this challenge, we designed a specific mental model for electronic questionnaires, which will be presented in Section 3.

\section{MENTAL MODEL}

To transfer paper-based questionnaires into electronic ones and to meet the requirements discussed, we designed a mental model for the support of mobile electronic questionnaires (cf. Figure 1). According to this model, the logic of a paper-based questionnaire is described in terms of a process model, which is then deployed to a process management system. The latter allows creating and executing process (i.e., questionnaire) instances.

Generally, a process model serves as template for specifying and automating well defined processes based on process management technology. In addition, adaptive process management systems allow for dynamic process changes of instances to handle unplanned exceptional situations as well (Reichert and Weber, 2012). In the following, we show that processawareness is useful for realizing applications other than business process automation as well. Applying the process paradigm and process management technology in the context of mobile data collection, however, raises additional challenges (cf. Section 5). We will show how to realize a process-aware approach that guides users in filling in electronic questionnaires based on process management technology.

\subsection{Process Model and Instances}

As opposed to traditional information systems, process-aware information systems (PAIS) separate process logic from application code. This is accomplished based on process models, which provide the schemes for executing the respective processes (Weber et al., 2011). In addition, a process model allows for a visual (i.e., graph-based) representation of the corresponding process, comprising activities (i.e., process steps) as well as the relations (i.e., control and data flow) between them. For control flow modeling, both control edges and gateways (e.g., ANDsplit, XORsplit) are provided.

A process model $P$ is represented as a directed, structured graph, which consists of a set of nodes $N$ (of different types $N T$ ) and directed edges $E$ (of different types $E T$ ) between them. We assume that a process model has exactly one start node $(N T=$ StartFlow) and one end node $(N T=E n d F l o w)$. Further, a process model must be connected; i.e., each node $n$ can be reached from the start node. In turn, from any node $n$ of a process model, the end node can be reached. In this paper, we solely consider blockstructured process models. Each branching (e.g. parallel or alternative branching) has exactly one entry and one exit node. Further, such blocks may be 


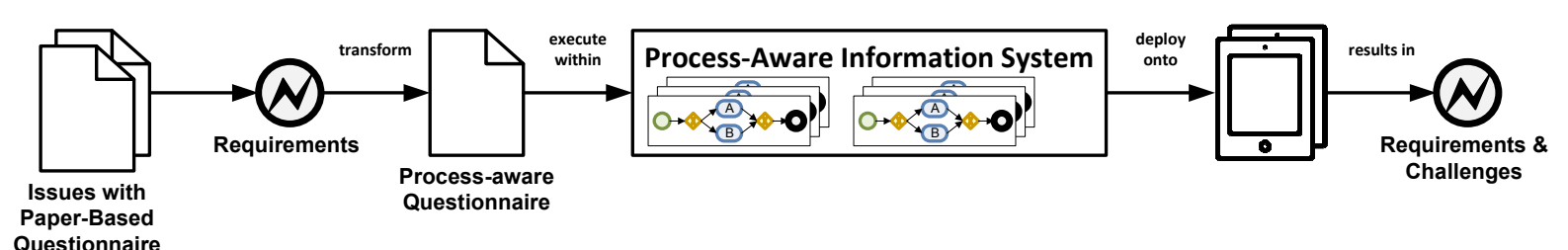

Figure 1: Mental model.

nested, but are not allowed to overlap (Reichert and Dadam, 2009). In turn, data elements $D$ correspond to global variables, which are connected with activities through data flow edges (ET DataFlow). These data elements can either be read (ReadAccess) or written (WriteAccess) by an activity (Reichert and Dadam, 1998) from the process model. Figure 3 shows an example of such a process model.

In turn, a process instance $I$ represents a concrete case that is executed based on a process model $P$. In general, multiple instances of a process model may be created and then concurrently executed. Thereby, the state of an instance is defined by the marking of its nodes and edges as well as the values of its data elements. Altogether, respective information corresponds to the execution history of an instance. The process engine has a set of execution rules which describe the conditions under which a node may be activated (Reichert and Dadam, 1998). If its end node is reached, a process instance terminates. An example of how to map a questionnaire to a process model is provided in Section 3.2.

\subsection{Mapping a Questionnaire to a Process Model}

Our mental model enabling a process-driven enactment of questionnaires is as follows: We define both the contents and the logic of a questionnaire in terms of a process model. Thereby, pages of the questionnaire logically correspond to process activities, whereas the flow between these activities specifies the logic of the questionnaire. The questions themselves are mapped to process data elements, which are connected with the respective activity. There are separate elements containing the text of a question, which can be read by the activity. Moreover, there are data elements that can be written by the activity. The latter are used to store the given answers for a specific question. Figure 2 gives an overview of the mapping of the elements of a questionnaire to the ones of a process model.

To illustrate the process-driven modeling of electronic questionnaires, we present a scenario from psychology. Consider the process-centric questionnaire model from Figure 3. Its process logic is described in

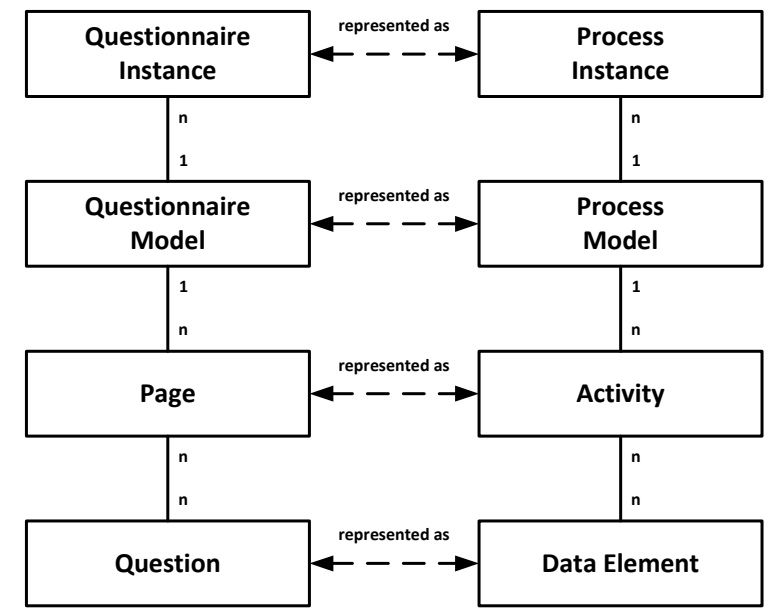

Figure 2: Mapping a Questionnaire Model to a Process Model.

terms of BPMN 2.0 (Business Process Model and Notation) (Business Process Model, 2011). To establish the link between process and questionnaire model, we annotated the depicted graph with additional labels.

The processing of the questionnaire starts with the execution of activity Page Intro, which presents an introductory text for the participant interacting with the electronic questionnaire. This introduction includes, for example, instructions on how to fill in the questionnaire or how to interact with the smart mobile device. After completing this first step, activity Page General becomes enabled. In this form-based activity, data elements Cigarettes, Drugs and Alcohol are written. More precisely, the values of these data elements correspond to the answers given for the questions displayed on the respective page of the questionnaire. For example, the question corresponding to data element Cigarettes is as follows: "Do you smoke?" (with the possible answers "yes / no"). After completing activity Page General, an AND gateway (ANDsplit) becomes enabled. In turn, all outgoing paths of this ANDsplit (i.e., parallel split node) become enabled and are then executed concurrently. In the given application scenario, each of these paths contains an XOR Gateway (XORsplit), which reads one of the aforementioned data elements to make a choice among its outgoing paths. For example, as- 


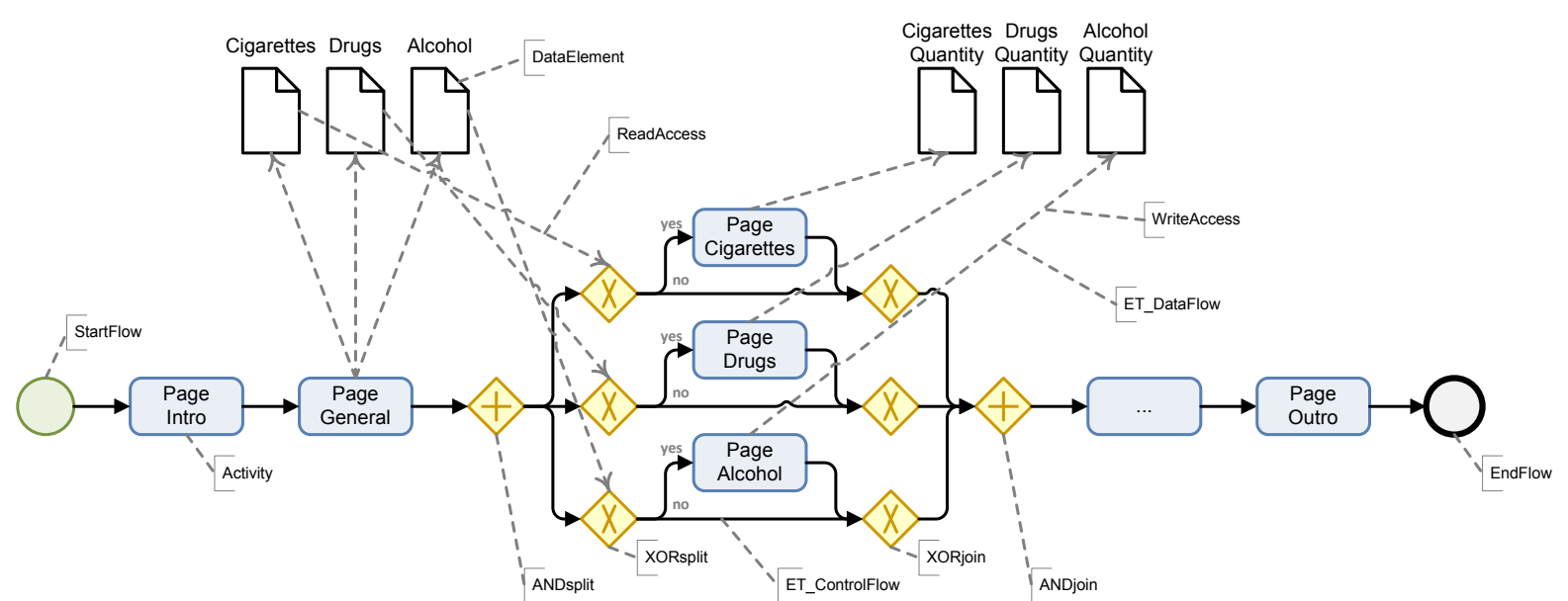

Figure 3: Application Scenario: an abbreviated Questionnaire with Annotations.

sume that in Page General the participant has answered question "Do you smoke?" with "yes". Then, in the respective XOR split, the upper path (labeled with "yes") will be chosen, which consists of exactly one activity, i.e., Page Cigarettes. In the context of this activity, additional questions regarding the consumption of cigarettes will be displayed to the actor. This activity and page, respectively, is exemplarily displayed in Figure 4. Assume further that question "Do you take drugs? (yes / no)" has been answered with "no" in the context of Page General. Then, activity Page Drugs will be skipped as the lower path (labeled with "no") of the respective XOR split will be chosen. As soon as all three branches are completed, the ANDjoin will become enabled and the succeeding activity be displayed. We omit further descriptions for activities of the questionnaire model due to lack of space. Finally, the processing of a questionnaire ends with activity Page Outro. Note that an arbitrary number of questionnaire instances processed by different participants may be created.

Figure 4 gives an impression of the Page Cigarettes activity. It displays additional questions regarding the consumption of cigarettes. This page is layouted automatically by the electronic questionnaire application based on the specified process model, which includes the pages to be displayed (cf. Figure 3 ). Note, that the data elements are used to create the user interface, as they contain the actual text of the questions as well as the possible answers to be displayed (i.e., the answers among which the user may choose).

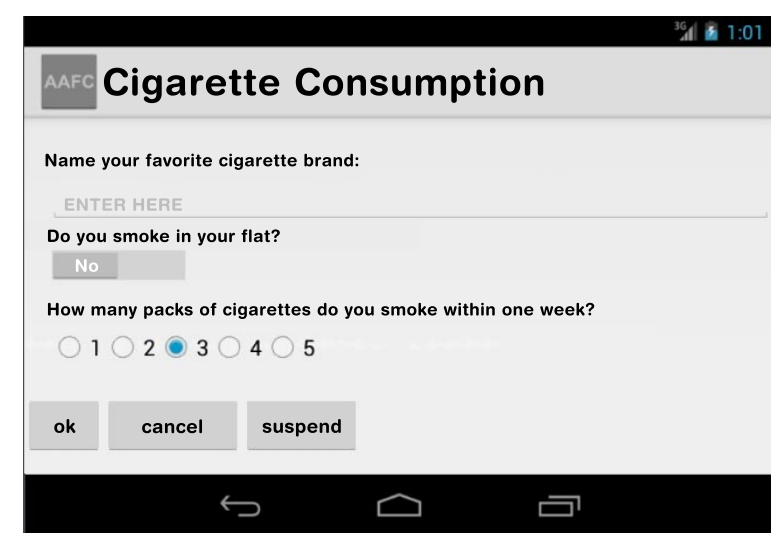

Figure 4: Activity "Page Cigarettes".

\subsection{Requirements for Process-Based Questionnaires}

When using process management technology to coordinate the collection of data with smart mobile devices, additional challenges emerge. In particular, these are related to the modeling of a questionnaire as well as the process-driven execution of corresponding questionnaire instances on smart mobile devices.

Since questionnaire-based interviews are often interactive, the participating roles (e.g., interviewer and interviewed subject) should be properly assisted when interacting with the smart mobile device. For example, it should be possible for them to start or abort questionnaire instances. In the context of longrunning questionnaire instances, in addition, it might be required to interrupt an interview and continue it later. For this purpose, it must be possible to suspend the execution of a questionnaire instance and to resume it at a later point in time (with access to all data and answers collected so far). In the context of 


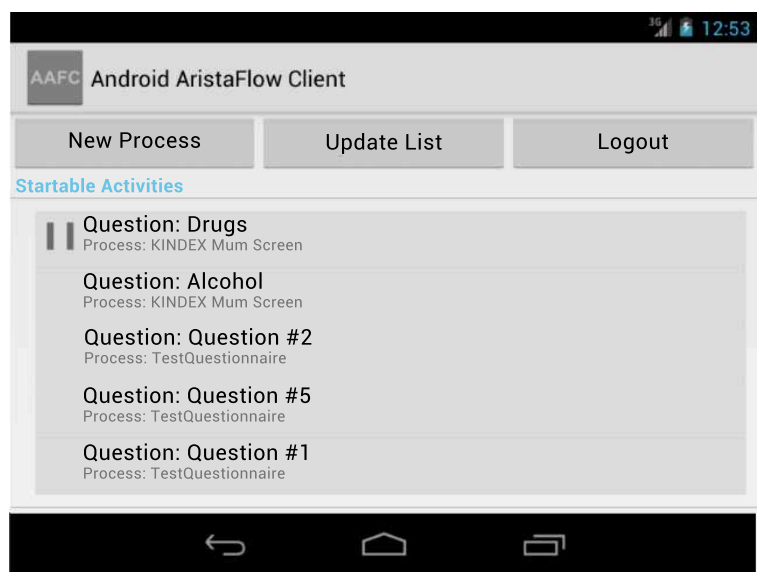

Figure 5: Startable Activities for a Specific Actor.

long-running interviews, it is also useful to be able to display an entire questionnaire and process model respectively. Therefore, already answered questions should be displayed differently (e.g., in a different color) compared to upcoming questions. Note that this is crucial for providing a quick overview about the progress of an interview.

Since domain experts might not be familiar with existing process modeling notations like BPMN 2.0, an easy-to-understand, self-explaining, and domainspecific process notation is needed. In addition, the roles participating in a questionnaire should be provided with specific views on the process model (i.e., questionnaire), e.g., hiding information not required for this role (Kolb and Reichert, 2013). For example, a subject may not be allowed to view subsequent questions in order to ensure credibility of the given answers.

Regarding the execution of the activities of a questionnaire (i.e., pages) additional challenges emerge.

The questions of a (psychological) questionnaire may have to be answered by different actors each of them possessing a specific role. For example, followup questions related to the subject involved in a psychological questionnaire might have to be answered by a psychologist and not by the subject itself. Consequently, the electronic questionnaire application must ensure that only those questions are displayed to an actor intended for him or her. Figure 5 shows the startable activities, currently available for a specific actor using the smart mobile device.

In many scenarios, the questions of an electronic questionnaire may have to be displayed together with possible answers. In order to avoid bad quality of the data collected, actors should be further assisted when interacting with the smart mobile device; e.g., through error messages, help texts, or on-the-fly validations of entered data.
To foster the subsequent analysis of the data collected, the latter needs to be archived in a central repository. Furthermore, additional information (e.g., the time it took the subject to answer a particular question) should be recorded in order to increase the expressiveness of the data collected. Finally, anonymization of this data might have to be ensured as questionnaires often collect personal data and privacy constitutes a crucial issue. In certain cases, it might also become neccessary to dismiss the results of an already answered question.

Taking these general requirements into account, we designed an architecture for an electronic questionnaire application.

\section{ARCHITECTURE AND IMPLEMENTATION}

This section introduces the architecture we developed for realizing mobile electronic questionnaires. In particular, the latter run on smart mobile devices and interact with a remote process engine. This architecture is presented in Section 4.1. Since this paper focuses on the requirements, challenges and lessons learned when applying state-of-the-art process management technology to realize electronic questionnaires, we will not describe the architecture of the process management system (and its process engine) in detail; see (Dadam and Reichert, 2009; Reichert et al., 2009) for respective work. The general architecture of our electronic questionnaire application is depicted in Figure 6.

\subsection{Electronic Questionnaire Application}

The electronic questionnaire application is divided into three main packages, which are related to the user interface (1), the communication (2) with the external process engine, and useful tools for interacting with the client (3).

The user interface representing a particular page of the questionnaire is represented by an ActivityTemplate (4), which provides basic methods for the questionnaire (e.g., to start or stop an activity). In turn, the LoginView (5) is used to query the user credential and to select an available role for this actor (e.g., name $=$ JohnDoe, role $=$ Interviewer $)$. Furthermore, the MainView (6) provides a list (e.g., worklist) with the pages currently available for the user interacting with the questionnaire. These list items are represented using the ProcessAdapter (7). Since the user 


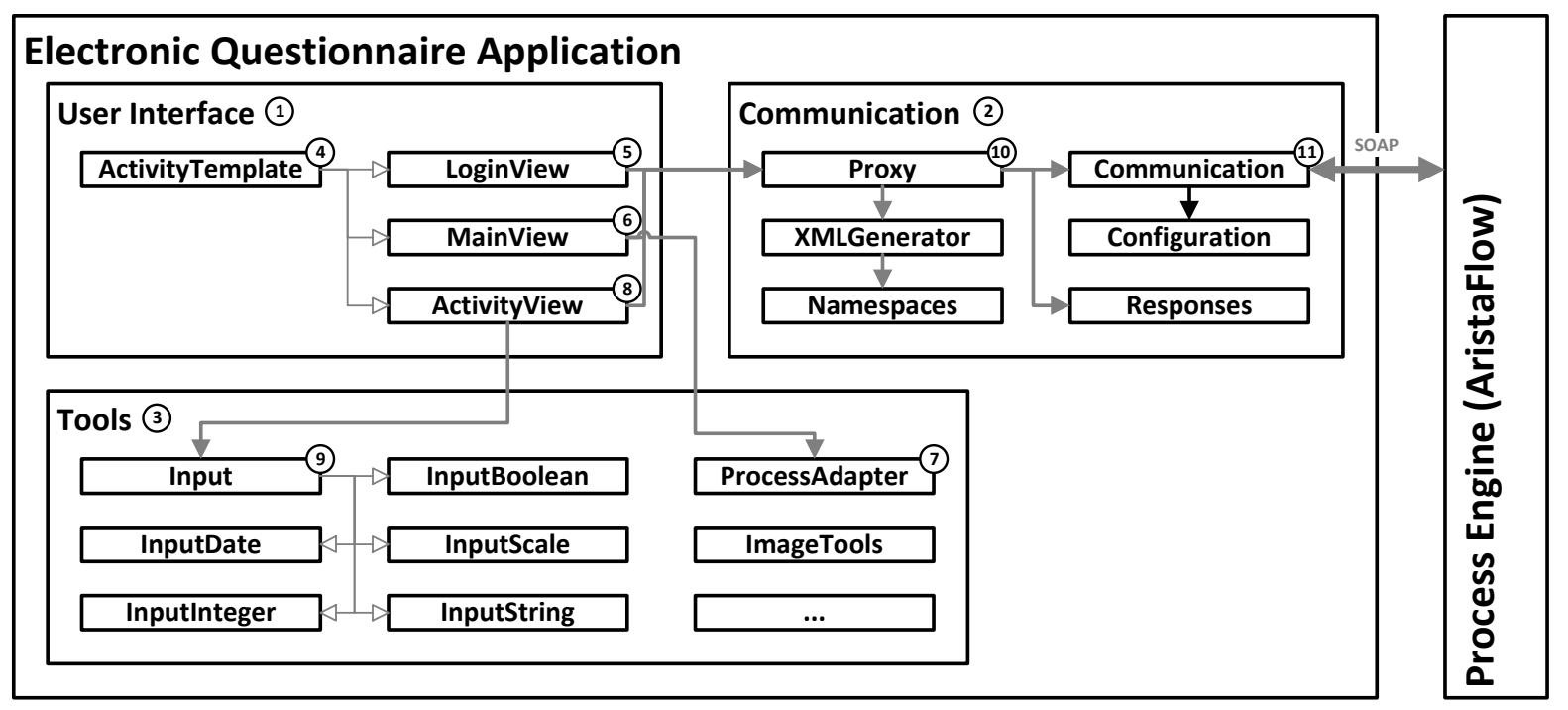

Figure 6: Architecture of the questionnaire application.

interface of a questionnaire is generated dynamically depending on the underlying process model deployed on the process engine at runtime, a user interface generator is needed. This service is provided by the ActivityView 88. To interact with the device, different classes of the Input (9) elements used within a questionnaire are provided. These classes provide the necessary logic to interact with the input elements as well as the corresponding graphical representation of this element. As certain input elements are platformspecific (e.g., there is no spinning wheel for standard desktop applications), missing input elements might be rendered differently, depending on the underlying platform (e.g., the spinning wheel on the iOS platform could be rendered as a dropdown element on a normal computer).

The complete communication with the external process engine should be handled by a Proxy (10) service. The latter is capable of generating the necessary request messages, which are then converted to SOAP request messages by the Communication (11) service and sent to the process engine. The response messages (e.g., the next page to display) sent by the process engine are then received by the Communication and decomposed by the Proxy. Afterwards, the data within this message is visualized in the ActivityView, which includes the already mentioned user interface generator as well.

\subsection{Proof-of-Concept Prototype}

To validate the feasibility of the described architecture as well as to be able to apply it in a real setting, we implemented a proof-of-concept prototype for the

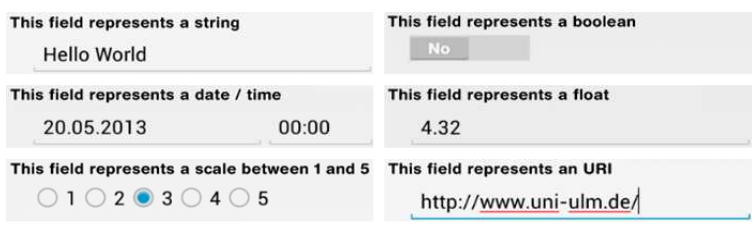

Figure 7: Different question types and their visualization within the questionnaire client application.

Android platform. We decided to use the latter, since it is easier to generate and handle SOAP (Curbera et al., 2002) calls within the application compared to iOS. The prototype application was then used to verify the prescribed mental model (cf. Section 3), and to detail the requirements regarding the execution of process-aware questionnaires. Furthermore, additional insights into the practical use of this electronic questionnaire application by domain experts in the context of their studies could be gathered. We were able to meet the requirements presented in Section 2.2 when implementing the questionnaire client application, even though certain drawbacks still exist. To enable domain experts, who usually have no programming skills, to create a mobile electronic questionnaire, we implemented a fully automated user interface generator for the mobile application itself. In addition, we were able to provide common types for questions used within a questionnaire (e.g., likertscale, free-text, or yes-no-switches). These types are automatically mapped to appropriate input elements visualized within the application. Figure 7 gives an impression of the input elements implemented. 


\section{DISCUSSION}

This section discusses our approach and reflects on the experiences we gained when applying state-ofthe-art process management technology to support mobile data collection with electronic questionnaires. Since we applied an implemented questionnaire in a psychological study, we were also able to gain additional insights into practical issues.

The presented approach has focused on the development of smart mobile device applications enabling flexible data collection rather than on the design of a development framework. Therefore, we have used an existing process modeling editor for defining the process logic of electronic questionnaires. However, since the domain experts using our questionnaire application have been unfamiliar with the BPMN 2.0 modeling notation, a number of training sessions were required to make them familiar with BPMN 2.0. Afterwards, they were able to create their own questionnaires. In particular, the abstraction introduced by the use of process models for specifying the logic of questionnaires was well understood by domain experts. However, the training sessions have shown that there is a need for a more user-friendly, domain-specific modeling notation, enabling domain experts to define questionnaires on their own. In particular, such a domain-specific modeling language needs to be selfexplaining and easy to use. Further, it should hide modeling elements not required in the given use case. Note that BPMN 2.0 provides many elements not needed for defining the logic of electronic questionnaires. Consider, for example, the AND-gateways, which allow modeling the parallel execution. Regarding the use case of mobile data collection, it does not matter which path is going to be evaluated first. On the other hand, elements used for modeling the questionnaire should have a meaningful and expressive representation. Thus, an activity should be represented as page-symbol to add more context-aware information to the questionnaire model.

As we further learned in our case study, the creation and maintenance of a questionnaire constitutes a highly interactive, flexible and iterative task. In general, the editing of already existing, but not yet published questionnaires, should be self-explaining. Basic patterns dealing with the adaptation of the logic of a questionnaire (e.g., moving a question to another position or adding a new question) should be integrated in a modeling editor to provide tool-support for creating and managing questionnaires.

As discussed in Section 3.3, we use process management technology for modeling and enacting electronic questionnaires. Accordingly the created ques- tionnaire model needs to be deployed on a process engine. Regarding the described client server architecture (cf. Section 4.1), all process (i.e., questionnaire) models are stored and executed on the server running the process engine. Keeping in mind that mobile questionnaires might be also used in areas without stable internet connection, any approach requiring a permanent internet connection between the mobile client and the process engine running on an external server will not be accepted. In order to cope with this issue, a light-weight process engine is required, which can run on the smart mobile device. We have started working in this direction as well; e.g., see (Pryss et al., 2010a; Pryss et al., 2010b).

Since the user interface of the electronic questionnaire is automatically generated based on the provided process model, the possibilities to customize the layout of a questionnaire are rather limited. From the feedback we had obtained from domain experts, however, it became clear that an expressive layout component is needed that allows controlling the visual appearance of a questionnaire running on smart mobile devices. Among others, different text styles (e.g., bold), spacing between input elements (e.g., bottom spacing), and absolute positioning of elements should be supported. In addition, the need for integrating images has been expressed several times.

Since we use process-driven electronic questionnaires for collecting data with smart mobile devices, the answers provided by the actors filling in the questionnaire could be directly transferred to the server. This will relieve the actors from time-consuming manual tasks. Furthermore, as there exists a process model describing the flow logic of the questionnaire as well as comprehensive instance data (e.g., instance execution history), process mining techniques for analyzing questionnaire instances may be applied (van der Aalst et al., 2007). In addition, Business Intelligence Systems (Anandarajan et al., 2003) could reveal further interesting aspects with respect to the data collected in order to increase the expressiveness of the analysis. Such systems would allow for a faster evaluation and relieve domain experts from manual tasks such as transferring the collected data into electronic worksheets.

Finally, we have experienced a strong acceptance among all participating actors (e.g., interviewers, domain experts, and subjects) regarding the practical benefits of electronic questionnaire applications on smart mobile devices. Amongst others this was reflected by a much higher willingness to fill out an electronic questionnaire compared to the respective paper-based version (Ruf-Leuschner et al., 2013; Isele et al., 2013). Furthermore, a higher motivation 
to complete the questionnaire truthfully could be observed. Of course, this acceptance partly results from the modern and intuitive way to interact with smart mobile devices.

\section{RELATED WORK}

There exists a variety of questionnaire systems available on the market. In general, these systems can be classified into two groups: online services (SurveyMonkey, 2013) and standalone applications (Electric Paper Evaluationssysteme, 2013). Due to the fact that a questionnaire might contain sensitive information (e.g., the mental status of a subject or personal details), online surveys are often not appropriate for this type of data collection applications. As another limitation of online systems, local authorities do often not allow third-party software systems to store the information of a subject. However, these applications also must deal with privacy issues. Standalone applications usually offer possibilities to create a questionnaire, but do not deal with the requirements discussed in this paper. Furthermore, they lack a flexible and mobile data collection. Usually, respective questionnaires are displayed as web applications, which cannot be used when no internet connection is available.

To the best of our knowledge, the process-aware enactment of questionnaires on smart mobile devices has not been considered comprehensively by other groups so far. In previous studies, we identified crucial issues regarding the implementation of psychological questionnaires on smart mobile devices (Liebrecht, 2012; Schindler, 2013). In these studies, we aimed at preserving the validity of psychological instruments, which is a crucial point when replacing paper-based questionnaires with electronic ones. Although the implemented applications have already shown several advantages in respect to data collection and analysis, they have not been fully suitable for realizing psychological questionnaires in the large scale yet. In particular, maintenance efforts for domain experts and other actors were considerably high. More precisely, changes of an implemented questionnaire (or its structure) still had to be accomplished by computer scientists, since its implementation is hardcoded. Therefore aim at the integration of a processaware information system to overcome this limitation.

Focusing on the complete lifecycle of paper-based questionnaires and supporting every phase with mobile technology has actually not been considered by other groups so far. However, there exists some work related to mobile data collection. In particular, mobile process management systems, as described in
(Pryss et al., 2013; Wakholi et al., 2011; Kunze et al., 2007), could be used to realize electronic questionnaires. However, this use case has not been considered by respective mobile process engines so far.

The QuON platform (Paul et al., 2013) provides a web-based survey system, which provides a variety of different input types for the questions used within a questionnaire. QuON does not use a model-based representation to specify a questionnaire as in our approach. Another distinctive characteristic of QuON is the webbased-only approach. Especially in psychological field studies, the latter will result in problems as the QuON platform does not use responsive webdesign.

Movilitas (Movilitas, 2013) applies SAP Sybase Unwired Platform to enable mobile data collection for business scenarios. The Sybase Unwired Platform is a highly adaptive implementation framework for mobile applications, which directly interacts with a backend, providing all required business data. Further research is required to show, whether this approach can be used to realize mobile electronic questionnaires in domains like psychology or health care as well.

Finally, (Kolb et al., 2012) present a set of patterns for for expressing user interface logic based on the same notation as used for business process modeling. In particular, a transformation method is introduced, which applies these patterns to automatically derive user interfaces by establishing a bidirectional mapping between process model and user interface.

\section{SUMMARY \& OUTLOOK}

In this paper, limitations of paper-based questionnaires for data collection were discussed. To deal with these limitations, we derived characteristic requirements for electronic questionnaire applications. In order to meet these requirements, we suggested the use of process management technology. According to the mental model introduced, a questionnaire and its logic can be described in terms of a process model at a higher level of abstraction. To evaluate our approach, a sophisticated application scenario from the psychological domain was considered. We have shown how a questionnaire can be mapped to a process model.

In the interviews we conducted with domain experts as well as from other implemented business applications we elaborated general requirements for flexible mobile data collection on smart mobile devices. These cover major aspects such as the secure and encrypted communication. Note that the latter is crucial, especially in the medical and psychological domains, which both deal with sensitive informa- 
tion of the subjects involved. We further presented an architecture enabling such mobile data collection applications based on a smart mobile device and a process engine. As another contribution, we demonstrated the feasibility of our proof-of-concept application. Several features as well as problems regarding the implementation and communication with the server component, hosting the process engine, have been highlighted. Finally, we discussed the benefits of using process-aware questionnaire application for mobile data collection.

In future work, we plan to extend our approach with additional features. First, we will provide a mobile process engine running on the smart mobile device itself. This will enable a process-driven enactment of questionnaire instances even if no permanent internet connection is available. We consider this as a fundamental feature for enabling flexible data collection applications on smart mobile devices. However, this will be accompanied with other problems, such as the proper synchronization among multiple devices (e.g., if changes were made to the model of the questionnaire) in order to keep the devices at the same level of information. In addition, we want to conceptualize a generic questionnaire system, which is able to support the complete lifecycle of a questionnaire. To disseminate this system among domain experts being unfamiliar and unaware of modeling process logic with standard notations, in addition, an easy to understand, but still precise notation for defining processaware questionnaires is needed. To further enhance data analysis capabilities (e.g., further analysis of the given answers), we have started to integrate sensors measuring vital signs in order to gather other information about subjects during interviews (Schobel et al., 2013). As a major benefit of the framework, we expect higher data quality, shorter evaluation cycles and a significant decrease in workload. In particular, it enables a high level of abstraction in defining electronic questionnaires that may run on smart mobile devices.

\section{Acknowledgement}

Supported by funds from the program Research initiatives, intrastructure, network and transfer plattforms in the framework of the DFG Excellence Initiative Third Funding Line.

\section{REFERENCES}

Anandarajan, M., Anandarajan, A., and Srinivasan, C. A. (2003). Business intelligence tech- niques: a perspective from accounting and finance. Springer.

Business Process Model (2011). Business Process Model and Notation (BPMN) Version 2.0. OMG Specification, Object Management Group.

Crombach, A., Nandi, C., Bambonye, M., Liebrecht, M., Pryss, R., Reichert, M., Elbert, T., and Weierstall, R. (2013). Screening for mental disorders in post-conflict regions using computer apps - a feasibility study from burundi. In XIII Congress of European Society of Traumatic Stress Studies (ESTSS) Conference.

Curbera, F., Duftler, M., Khalaf, R., Nagy, W., Mukhi, N., and Weerawarana, S. (2002). Unraveling the Web services web: an introduction to SOAP, WSDL, and UDDI. IEEE, Internet Computing, 6(2):86-93.

Dadam, P. and Reichert, M. (2009). The ADEPT Project: A Decade of Research and Development for Robust and Flexible Process Support Challenges and Achievements. Computer Science - Research and Development, 23(2):81-97.

Electric Paper Evaluationssysteme (2013). EvaSys. http://www.evasys.de/. last visited: 05. November 2013.

Isele, D., Ruf-Leuschner, M., Pryss, R., Schauer, M., Reichert, M., Schobel, J., Schindler, A., and Elbert, T. (2013). Detecting adverse childhood experiences with a little help from tablet computers. In XIII Congress of European Society of Traumatic Stress Studies (ESTSS) Conference.

Kolb, J., Hübner, P., and Reichert, M. (2012). Automatically Generating and Updating User Interface Components in Process-Aware Information Systems. In 20th Int'l Conference on Cooperative Information Systems, number 7565 in LNCS, pages 444-454. Springer.

Kolb, J. and Reichert, M. (2013). A flexible approach for abstracting and personalizing large business process models. Applied Computing Review, 13(1):6-17.

Kunze, C. P., Zaplata, S., and Lamersdorf, W. (2007). Mobile processes: Enhancing cooperation in distributed mobile environments. Journal of Computers, 2(1):1-11.

Liebrecht, M. (2012). Technische Konzeption und Realisierung einer mobilen Anwendung für den Konstanzer Index zur Erhebung von psychosozialen Belastungen während der Schwangerschaft. Diploma Thesis, University of Ulm.

Movilitas (2013). Movilitas Consulting AG. 
http://www.movilitas.com/. last visited: 04 . November 2013.

Paul, D., Wallis, M., Henskens, F., and Nolan, K. (2013). QuON: A Generic Platform for the Collation and Sharing of Web Survey Data. International Conference on Web Information Systems and Technologies.

Pryss, R., Langer, D., Reichert, M., and Hallerbach, A. (2012). Mobile Task Management for Medical Ward Rounds - The MEDo Approach. In 1st Int'l Workshop on Adaptive Case Management (ACM'12), BPM'12 Workshops, number 132 in LNBIP, pages 43-54. Springer.

Pryss, R., Musiol, S., and Reichert, M. (2013). Collaboration Support Through Mobile Processes and Entailment Constraints. In 9th IEEE Int'l Conference on Collaborative Computing: Networking, Applications and Worksharing. IEEE Computer Society Press.

Pryss, R., Tiedeken, J., Kreher, U., and Reichert, M. (2010a). Towards flexible process support on mobile devices. In Proc CAiSE'10 Forum - Information Systems Evolution, number 72 in LNBIP, pages 150-165. Springer.

Pryss, R., Tiedeken, J., and Reichert, M. (2010b). Managing Processes on Mobile Devices: The MARPLE Approach. In CAiSE'10 Demos.

Reichert, M. and Dadam, P. (1998). ADEPTflexSupporting Dynamic Changes of Workflows Without Losing Control. Journal of Intelligent Information Systems, Special Issue on Workflow Management Systems, 10(2):93-129.

Reichert, M. and Dadam, P. (2009). Enabling Adaptive Process-aware Information Systems with ADEPT2. In Cardoso, J. and van der Aalst, W., editors, Handbook of Research on Business Process Modeling, pages 173-203. Information Science Reference, Hershey, New York.

Reichert, M., Dadam, P., Rinderle-Ma, S., Jurisch, M., Kreher, U., and Goeser, K. (2009). Architecural Principles and Components of Adaptive Process Management Technology. In PRIMIUM - Process Innovation for Enterprise Software, number P-151 in Lecture Notes in Informatics (LNI), pages 81-97.

Reichert, M. and Weber, B. (2012). Enabling Flexibility in Process-Aware Information Systems: Challenges, Methods, Technologies. Springer, Berlin-Heidelberg.

Robecke, A., Pryss, R., and Reichert, M. (2011). DBIScholar: An iPhone Application for Performing Citation Analyses. In CAiSE Forum-
2011, volume Vol-73 of Proc CAiSE'11 Forum. CEUR Workshop Proceedings.

Ruf-Leuschner, M., Pryss, R., Liebrecht, M., Schobel, J., Spyridou, A., Reichert, M., and Schauer, M. (2013). Preventing further trauma: KINDEX mum screen - assessing and reacting towards psychosocial risk factors in pregnant women with the help of smartphone technologies. In XIII Congress of European Society of Traumatic Stress Studies (ESTSS) Conference.

Schindler, A. (2013). Technische Konzeption und Realisierung des MACE-Tests mittels mobiler Technologie. Bachelor Thesis, University of Ulm.

Schobel, J., Schickler, M., Pryss, R., Nienhaus, H., and Reichert, M. (2013). Using Vital Sensors in Mobile Healthcare Business Applications: Challenges, Examples, Lessons Learned. In 9th Int'l Conference on Web Information Systems and Technologies (WEBIST 2013), Special Session on Business Apps, pages 509-518.

SurveyMonkey (2013). SurveyMonkey: Free online survey software \& questionnaire tool. http://www.surveymonkey.com/. last visited: 14 . May 2013.

van der Aalst, W. M., Reijers, H. A., Weijters, A. J., van Dongen, B. F., Alves de Medeiros, A., Song, M., and Verbeek, H. (2007). Business process mining: An industrial application. Information Systems, 32(5):713-732.

Wakholi, P., Chen, W., and Klungsøyr, J. (2011). Workflow support for mobile data collection. In Enterprise, Business-Process and Information Systems Modeling, pages 299-313. Springer.

Weber, B., Reichert, M., Mendling, J., and Reijers, H. (2011). Refactoring large process model repositories. Computers in Industry, 62(5):467-486. 